PROCEEDINGS OF THE

AMERICAN MATHEMATICAL SOCIETY

Volume 135, Number 6, June 2007, Pages 1839-1842

S 0002-9939(06)08671-0

Article electronically published on December 29, 2006

\title{
MINIMAL RANK AND REFLEXIVITY OF OPERATOR SPACES
}

\author{
ROY MESHULAM AND PETER ŠEMRL
}

(Communicated by Joseph A. Ball)

\begin{abstract}
Let $\mathcal{S}$ be an $n$-dimensional space of linear operators between the linear spaces $U$ and $V$ over an algebraically closed field $\mathbb{F}$. Improving results of Larson, Ding, and Li and Pan we show the following.

Theorem. Let $S_{1}, \ldots, S_{n}$ be a basis of $\mathcal{S}$. Assume that every nonzero operator in $\mathcal{S}$ has rank larger than $n$. Then a linear operator $T: U \rightarrow V$ belongs to $\mathcal{S}$ if and only if for every $u \in U, T u$ is a linear combination of $S_{1} u, \ldots, S_{n} u$.
\end{abstract}

\section{INTRODUCTION}

Let $U$ and $V$ be linear spaces over a field $\mathbb{F}$, and let $\mathcal{L}(U, V)$ denote the space of all linear operators from $U$ to $V$. Let $\mathcal{S} \subset \mathcal{L}(U, V)$ be a linear subspace of operators. An operator $T \in \mathcal{L}(U, V)$ is locally linearly dependent on $\mathcal{S}$, if $T u \in \mathcal{S} u=\{S u: S \in \mathcal{S}\}$ for all $u \in U$. The reflexive closure of $\mathcal{S}$ is the space $\operatorname{ref}(\mathcal{S})$ of all $T \in \mathcal{L}(U, V)$ that are locally linearly dependent on $\mathcal{S}$.

The space $\mathcal{S}$ is (algebraically) reflexive if $\operatorname{ref}(\mathcal{S})=\mathcal{S}$. Various aspects of reflexive operator spaces were studied in [5, 6, 7, 8, 10, In this paper we are concerned with the relation between minimal rank and reflexivity. Let

$$
\rho(\mathcal{S})=\min \{\operatorname{rank}(A): 0 \neq A \in \mathcal{S}\}
$$

and let

$$
f_{\mathbb{F}}(n)=\sup \{\rho(\mathcal{S}): \operatorname{dim} \mathcal{S}=n, \operatorname{ref}(\mathcal{S}) \neq \mathcal{S}\} .
$$

Larson 7] proved that if $\mathcal{S}$ is nonreflexive, then $\rho(\mathcal{S})<\infty$. Ding [6] gave a quantitative version of Larson's result by showing that $f_{\mathbb{F}}(n) \leq n^{2}$. This was further improved in the complex case by Li and Pan 8 , who proved $f_{\mathbb{C}}(n) \leq 2 n-2$. In [10] it was shown that if $|\mathbb{F}| \geq n+3$, then any $n$-dimensional nonreflexive space either contains a nonzero operator of rank $\leq 2 n-3$, or all of its nonzero members have rank $2 n-2$. In particular $f_{\mathbb{F}}(n) \leq 2 n-2$.

The study of reflexivity of finite-dimensional operator spaces is closely related to questions concerning locally linearly dependent operator spaces. An $n$-dimensional subspace $\mathcal{R} \subset \mathcal{L}(U, V)$ is locally linearly dependent if $\operatorname{dim} \mathcal{R} u<n$ for all $u \in U$. Locally linearly dependent spaces and reflexive spaces have many applications in operator theory and in ring theory (see e.g. 1, 4, 9]). In [9] it was shown that such

Received by the editors April 7, 2005 and, in revised form, February 10, 2006.

2000 Mathematics Subject Classification. Primary 47L05; Secondary 15A03.

The research of the first author was supported in part by the Israel Science Foundation.

The research of the second author was supported in part by a grant from the Ministry of Science of Slovenia.

(C)2006 American Mathematical Society Reverts to public domain 28 years from publication 
$\mathcal{R}$ must contain a nonzero operator of rank at most $n-1$. Let $\mathcal{S}$ be a nonreflexive $n$-dimensional space of operators and assume that $T \in \operatorname{ref}(\mathcal{S})-\mathcal{S}$. Then clearly $\mathcal{R}=\operatorname{Span}\{T\}+\mathcal{S}$ is locally linearly dependent. In [10] we used this fact together with a structural result for minimal locally linearly dependent operator spaces to obtain the above-mentioned $f_{\mathbb{F}}(n) \leq 2 n-2$ bound. However, one cannot expect this approach to yield sharp estimates, since the condition $T \in \operatorname{ref}(\mathcal{S})-\mathcal{S}$ is in general stronger than local linear dependence of $\operatorname{Span}\{T\}+\mathcal{S}$. In fact, we conjecture that every $n$-dimensional nonreflexive space contains a nonzero operator of rank at most $n$. Our main result establishes this conjecture for algebraically closed fields.

Theorem 1.1. If $\mathbb{F}$ is algebraically closed, then

$$
\left\lfloor\frac{n}{2}-\sqrt{n}\right\rfloor \leq f_{\mathbb{F}}(n) \leq n .
$$

The upper bound in Theorem 1.1 is proved in section 2. In section 3 we prove the lower bound, and in addition give a simple construction which in particular shows that if $\mathbb{F}$ is finite or $\mathbb{F}=\mathbb{Q}$, then $f_{\mathbb{F}}(n) \geq n$.

\section{THE UPPER BOUND}

Let $\mathbb{F}$ be algebraically closed and let $\mathcal{S}$ be an $n$-dimensional subspace of $\mathcal{L}(U, V)$ such that $\operatorname{ref}(\mathcal{S}) \neq \mathcal{S}$. We have to show that there exists an $0 \neq S \in \mathcal{S}$ such that $\operatorname{rank} S \leq n$. By standard considerations (see e.g. Proposition 2.1 in 9]) we may assume that $U$ and $V$ are finite dimensional. We thus write $U=\mathbb{F}^{k}, V=\mathbb{F}^{\ell}$ and identify $\mathcal{L}(U, V)$ with $M_{\ell \times k}(\mathbb{F})$ - the space of $\ell \times k$ matrices over $\mathbb{F}$. Let $S_{1}, \ldots, S_{n}$ be a basis of $\mathcal{S}$. Let $u=\left(u_{1}, \ldots, u_{k}\right)$ be a vector of $k$ independent variables and let $\mathbb{K}$ denote the function field $\mathbb{F}\left(u_{1}, \ldots, u_{k}\right)$. For a column vector $v \in \mathbb{K}^{\ell}$ and a row vector $w \in \mathbb{K}^{k}$ we write $v \otimes w$ for the matrix product $v \cdot w \in M_{\ell \times k}(\mathbb{K})$.

Choose an operator $T \in \operatorname{ref}(\mathcal{S})-\mathcal{S}$. If $\operatorname{dim} \mathcal{S} a<\operatorname{dim} \mathcal{S}=n$ for all $a \in \mathbb{F}^{k}$, then by $[3,9, \mathcal{S}$ contains a nonzero matrix of rank at most $n-1$. We thus assume that $\operatorname{dim} \mathcal{S} a=n$ for some $a \in \mathbb{F}^{k}$. This implies that $S_{1} u, \ldots, S_{n} u$ are linearly independent vectors in $\mathbb{K}^{\ell}$. Since $T a \in \operatorname{Span}\left\{S_{1} a, \ldots, S_{n} a\right\}$ for all $a \in \mathbb{F}^{k}$, it follows (by the infinitude of $\mathbb{F}$ ) that $T u, S_{1} u, \ldots, S_{n} u$ are linearly dependent in $\mathbb{K}^{\ell}$. Thus, by Cramer's rule, there exist homogeneous polynomials $p(u), p_{1}(u), \ldots, p_{n}(u)$ of the same degree $d$, such that $p(u) \neq 0$ and such that

$$
p(u) T u=\sum_{i=1}^{n} p_{i}(u) S_{i} u .
$$

We may assume that g.c.d. $\left(p(u), p_{1}(u), \ldots, p_{n}(u)\right)=1$. Furthermore, $T \notin \mathcal{S}$ implies that $d \geq 1$. Differentiating (2) we obtain

$$
p(u) T+T u \otimes d p(u)=\sum_{i=1}^{n} p_{i}(u) S_{i}+\sum_{i=1}^{n} S_{i} u \otimes d p_{i}(u),
$$

where $d f(u)=\left(\frac{\partial f}{\partial u_{1}}(u), \ldots, \frac{\partial f}{\partial u_{k}}(u)\right)$. Therefore

$$
p(u) T-\sum_{i=1}^{n} p_{i}(u) S_{i}=\sum_{i=1}^{n} S_{i} u \otimes\left(d p_{i}(u)-\frac{p_{i}(u)}{p(u)} d p(u)\right) .
$$


The right-hand side is a sum of at most $n$ rank one matrices in $M_{\ell \times k}(\mathbb{K})$, hence

$$
\operatorname{rank}\left(p(u) T-\sum_{i=1}^{n} p_{i}(u) S_{i}\right) \leq n .
$$

If $\left(p_{1}(a), \ldots, p_{n}(a)\right)=0$ for all $a \in \mathbb{F}^{k}$ such that $p(a)=0$, then by the Nullstellensatz, $p(u)$ must divide some power of each of the $p_{i}(u)$ 's. Since $d \geq 1$, this implies that $p(u), p_{1}(u), \ldots, p_{n}(u)$ have a nontrivial common factor, a contradiction. Therefore, there exists an $a \in \mathbb{F}^{k}$ such that $p(a)=0$ but $\left(p_{1}(a), \ldots, p_{n}(a)\right) \neq 0$. By (5)

$$
0<\operatorname{rank}\left(\sum_{i=1}^{n} p_{i}(a) S_{i}\right) \leq n
$$

\section{THE LOWER BOUND}

We shall need a characterization of reflexivity due essentially to Azoff [2]. Consider the standard bilinear form on $M_{\ell \times k}(\mathbb{F})$ given by $(A, B)=\operatorname{tr}\left(A B^{T}\right)$. For a subspace $S \subset M_{\ell \times k}(\mathbb{F})$ let

$$
\mathcal{S}^{\perp}=\left\{B \in M_{\ell \times k}(\mathbb{F}):(A, B)=0 \text { for all } A \in \mathcal{S}\right\} .
$$

Let $R_{d}=\left\{A \in M_{\ell \times k}(\mathbb{F}): \operatorname{rank} A \leq d\right\}$. The following result is a variant of Proposition 2.2 in [2].

Claim 3.1. For any subspace $\mathcal{S} \subset M_{\ell \times k}(\mathbb{F})$

$$
\operatorname{ref}(\mathcal{S}) / \mathcal{S} \cong \mathcal{S}^{\perp} / \operatorname{Span}\left(\mathcal{S}^{\perp} \cap R_{1}\right)
$$

In particular, $\mathcal{S}$ is reflexive iff $\mathcal{S}^{\perp}$ is generated by rank one elements.

Proof. For $u \in \mathbb{F}^{k}$ let $z(u)=\left\{A \in M_{\ell \times k}(\mathbb{F}): A u=0\right\}$. Clearly $z(u)=\mathbb{F}^{\ell} \otimes u^{\perp}$. It follows that

Therefore

$$
\operatorname{ref}(\mathcal{S})=\bigcap_{u \in \mathbb{F}^{k}}(\mathcal{S}+z(u))=\bigcap_{u \in \mathbb{F}^{k}}\left(\mathcal{S}+\mathbb{F}^{\ell} \otimes u^{\perp}\right)
$$

$$
\begin{aligned}
\operatorname{ref} & (\mathcal{S})^{\perp}=\sum_{u \in \mathbb{F}^{k}}\left(\mathcal{S}+\mathbb{F}^{\ell} \otimes u^{\perp}\right)^{\perp} \\
& =\sum_{u \in \mathbb{F}^{k}}\left(\mathcal{S}^{\perp} \cap \mathbb{F}^{\ell} \otimes \operatorname{Span}\{u\}\right)=\operatorname{Span}\left(\mathcal{S}^{\perp} \cap R_{1}\right) .
\end{aligned}
$$

It follows that

$$
\mathcal{S}^{\perp} / \operatorname{Span}\left(\mathcal{S}^{\perp} \cap R_{1}\right)=\mathcal{S}^{\perp} / \operatorname{ref}(\mathcal{S})^{\perp} \cong \operatorname{ref}(\mathcal{S}) / \mathcal{S}
$$

Let $\mathbb{F}$ be algebraically closed. To prove the lower bound in Theorem 1.1, we show that there exists an $n$-dimensional nonreflexive operator space $\mathcal{S}$ such that $\rho(\mathcal{S}) \geq\left\lfloor\frac{n}{2}-\sqrt{n}\right\rfloor=r$. Let $k=\left\lfloor\frac{n}{2}\right\rfloor$ and let $\mathcal{S}$ be a generic $n$-dimensional subspace of $M_{k}(\mathbb{F})$. Recall that $R_{d}=\left\{A \in M_{k}(\mathbb{F}): \operatorname{rank} A \leq d\right\}$ is an irreducible affine variety of codimension $(k-d)^{2}$ in $M_{k}(\mathbb{F})$. Since

$$
\operatorname{dim} \mathcal{S}^{\perp}=k^{2}-n<(k-1)^{2}=\operatorname{codim} R_{1}
$$

it follows that $\mathcal{S}^{\perp} \cap R_{1}=0$, hence $\mathcal{S}$ is nonreflexive by Claim 3.1.

On the other hand

$$
\operatorname{dim} \mathcal{S}=n<(k-r+1)^{2}=\operatorname{codim} R_{r-1} .
$$

Hence $\rho(\mathcal{S}) \geq r$. 
For certain nonalgebraically closed fields, such as $\mathbb{F}=\mathbb{F}_{q}$ or $\mathbb{F}=\mathbb{Q}$, the lower bound can be significantly improved.

Claim 3.2. Let $n>1$ and suppose there exists a (not necessarily associative) $n$-dimensional division algebra $A$ over the field $\mathbb{F}$. Then $f_{\mathbb{F}}(n) \geq n$.

Proof. For $a \in A$ let $T_{a} \in \mathcal{L}(A)$ be given by $T_{a}(b)=a b$. Let $\mathcal{S}=\left\{T_{a}: a \in A\right\} \subset$ $\mathcal{L}(A)$. Clearly $\rho(\mathcal{S})=\operatorname{dim} \mathcal{S}=n$. On the other hand $\mathcal{S} b=A$ for all $0 \neq b \in A$ implies that $\operatorname{ref}(\mathcal{S})=\mathcal{L}(A)$, hence $\mathcal{S}$ is nonreflexive.

\section{REFERENCES}

1. S.A. Amitsur, Generalized polynomial identities and pivotal monomials, Trans. Amer. Math. Soc. 114 (1965), 210-226. MR0172902 (30:3117)

2. E.A. Azoff, On finite rank operators and preannihilators, Mem. Amer. Math. Soc. 64 No. 357 (1986), 1-85. MR0858467 (88a:47041)

3. M. Brešar and P. Semrl, On locally linearly dependent operators and derivations, Trans. Amer. Math. Soc. 351 (1999), 1257-1275. MR1621729 (99e:47039)

4. M. Brešar and P. Šemrl, Elementary operators as Lie homomorphisms or commutativity preservers, Proc. Edinburgh Math. Soc. 48 (2005), 37-49. MR.2117710 (2005k:16041)

5. L. Ding, Separating vectors and reflexivity, Linear Algebra Appl. 174 (1992), 37-52. MR.1176449 (94a:47075)

6. L. Ding, On a pattern of reflexivity of operator spaces, Proc. Amer. Math. Soc. 124 (1996), 3101-3108. MR:1343689 (97h:47039)

7. D.R. Larson, Reflexivity, algebraic reflexivity and linear interpolation, Amer. J. Math. 283 (1988), 283-299. MR0935008 (89d:47096)

8. J. Li and Z. Pan, Reflexivity and hyperreflexivity of operator spaces, J. Math. Anal. Appl. 279 (2003), 210-215. MR1970501 (2004a:47001)

9. R. Meshulam and P. Šemrl, Locally linearly dependent operators, Pacific. J. Math. 203 (2002), 441-459. MR1897909 (2003a:47005)

10. R. Meshulam and P. Semrl, Locally linearly dependent operators and reflexivity of operator spaces, Linear Algebra Appl. 383 (2004), 143-150. MR2073900 (2005g:47138)

Department of Mathematics, Technion, Haifa 32000, Israel

E-mail address: meshulam@math.technion.ac.il

Department of Mathematics, University of Ljubljana, Jadranska 19, SI-1000 LjubLJANA, SlOVENIA

E-mail address: peter.semrl@fmf.uni-lj.si 